\title{
Pacific
}

Journal of

Mathematics

\section{DEHN FUNCTIONS OF GROUPS AND EXTENSIONS OF COMPLEXES}

\author{
STEPHEN GARY BRICK
}




\title{
DEHN FUNCTIONS OF GROUPS AND EXTENSIONS OF COMPLEXES
}

\author{
STEPHEN G. BRICK
}

\begin{abstract}
We study extensions of two-complexes and the Dehn functions (i.e. the isoperimetric inequalities) of their fundamental groups.

If $A \subset B$ are two complexes and their quotient $X$ is diagramatically reducible then we obtain an upper bound for the Dehn function of $\pi_{1}(B)$ in terms of the Dehn functions of $\pi_{1}(A)$ and $\pi_{1}(X)$. In particular, we show that if the Dehn functions of $\pi_{1}(A)$ and $\pi_{1}(X)$ are bounded above by polynomials of degree $n$ and $m$, then the Dehn function of $\pi_{1}(B)$ is bounded above by a polynomial of degree $n \cdot m$.
\end{abstract}

0. Introduction. In this paper, we look at extensions of twocomplexes and the Dehn functions of their fundamental groups. We start by recalling some definitions from [Br1] (in addition, see [Gr], [Ge2], and [CEHLPT]). Let $K$ be a finite two-complex. We will consider edge-paths (i.e. cellular maps of intervals into $K$ ) and will use $|*|$ for the length function. Write $N_{K}$ for the set of edge-circuits that are null-homotopic in $K$. Given an integer $l \geq 0$, we set

$$
N_{K}(l)=\left\{w \in N_{K}|| w \mid \leq l\right\} .
$$

If $w \in N_{K}(l)$ then there is a Van-Kampen diagram for $w$, i.e. a pair $\mathbb{D}=(D, j)$ where $j: D \rightarrow K$ is a cellular map and $D$ is a simply connected finite planar complex with boundary cycle $\partial \mathbb{D}$ mapping to $w$. Note that the boundary cycle $\partial \mathbb{D}$ is different from the topological boundary. In particular, as some edges appear twice, the length of the boundary cycle may be larger than the number of edges in the boundary.

Let $a(\mathbb{D})$ (also written $a(D)$ ) be the combinatorial area of $\mathbb{D}$, i.e. the number of faces of $D$. Set

$$
\Delta_{K}(w)=\min \{a(\mathbb{D}) \mid \mathbb{D} \text { is a diagram for } w\} .
$$

We will say that a diagram $\mathbb{D}$ for $w$ is minimal if $\Delta_{K}(w)=a(\mathbb{D})$. The Dehn function of $K$ is the map $\delta_{K}: \mathbb{N} \rightarrow \mathbb{N}$, where $\mathbb{N}=\{0,1,2, \ldots\}$, defined by

$$
\delta_{K}(l)=\max \left\{\Delta_{K}(w) \mid w \in N_{K}(l)\right\} .
$$


Observe that $\delta_{K}$ is monotone increasing. If $\mathscr{P}$ is a finite presentation then its Dehn function is $\delta_{\mathscr{K}(\mathscr{P})}$ where $\mathscr{K}(\mathscr{P})$ is the associated twocomplex. This may also be formulated algebraically; see [Br1].

Given $f, g: \mathbb{N} \rightarrow \mathbb{N}$, we write $f \preccurlyeq g$ if there are constants $c_{1}, c_{2}$, and $c_{3}$ with

$$
f(l) \leq c_{1} l+c_{2} \cdot g\left(c_{3} l\right)
$$

for all $l \in \mathbb{N}$. We write $f \equiv g$, and say that $f$ and $g$ are of the same type, if both $f \preccurlyeq g$ and $g \preccurlyeq f$. Observe that if $f \preccurlyeq g$ and $g$ is bounded above by a polynomial of degree $m$, with $m \geq 1$, then so is $f$.

In [Br1] we proved the following result (also see [Al], [CEHLPT], and [Ge2]):

Proposition. If $K$ and $L$ are connected finite two-complexes with isomorphic fundamental groups then $\delta_{K} \equiv \delta_{L}$.

So the type of Dehn function depends only on the fundamental group. If $\pi_{1}(K)=G$, where $K$ is a finite connected two-complex, and if $\delta_{K}$ is bounded above by a linear, quadratic, etc. function then we say that $G$ satisfies a linear, quadratic, etc. isoperimetric inequality. Moreover, we abuse notation slightly and refer to $\delta_{K}$ as the Dehn function of $G$ (strictly speaking the Dehn function is a $\equiv$-class).

Of particular interest is when the Dehn function is bounded by a polynomial. Recall (see [Gr]) that a group is hyperbolic iff its Dehn function is bounded above by a linear function.

Associate a degree $\geq 1$ to $G$, called the Dehn degree of $G$, where $\operatorname{deg}(G) \leq n$, for $n \geq 1$, provided $\delta_{K} \preccurlyeq l^{n}$, where $K$ is a connected finite complex with fundamental group $G$.

We find it convenient to work in the combinatorial category. A cellular map is combinatorial provided it preserves dimension, while a complex is combinatorial if each attaching map, after a subdivision of its domain, is combinatorial. Given a pair of combinatorial complexes $A \subset B$, the combinatorial quotient, $B / / A$, is the quotient of $B / A$ gotten by "adjusting" its attaching maps to make them combinatorial (see [Ge1]). For example, if $A=\mathscr{K}\left\langle x \mid x^{2}\right\rangle$ and $B=\mathscr{K}\left\langle x, y, z \mid x^{2},[x y, z]\right\rangle$ then their combinatorial quotient is $X=\mathscr{K}\langle y, z \mid[y, z]\rangle$.

A combinatorial extension is a sequence of finite combinatorial complexes $\mathscr{E}: A \subset B \rightarrow B / / A$. Observe that as $B / / A$ is combinatorial no cell of $B \backslash A$ can have its boundary lying entirely in $A$. 
We will say that a combinatorial extension $\mathscr{E}: A \subset B \rightarrow B / / A$ is acceptable if the following holds:

(1) $A$ is $\pi_{1}$-injective in $B$, i.e. if $x \in A$ then $i_{*}: \pi_{1}(A, x) \rightarrow$ $\pi_{1}(B, x)$ is injective, where $i: A \subset B$,

(2) $B / / A$ has no redundant faces, i.e. distinct two-cells have distinct attaching maps.

We will write $\mathscr{A}(X)$ for the set of acceptable extensions $A \subset B \rightarrow$ $B / / A$ with $B / / A=X$. Regarding $\mathscr{A}(X)$, observe that condition (2) in the definition above is actually a condition on $X$. So $\mathscr{A}(X)$ being non-empty implies that $X$ has no redundant faces. Also, as we are only considering extensions of finite complexes, $X$ must itself be finite.

A function $g: \mathbb{N} \rightarrow \mathbb{N}$ is said to be subnegative if

$$
g(n)+g(m) \leq g(n+m)
$$

for all $n, m \in \mathbb{N}$. Given $f: \mathbb{N} \rightarrow \mathbb{N}$, the least subnegative function greater than or equal to $f$ is called the subnegative closure of $f$, denoted by $\mathscr{S}(f)$. It is given by the formula

$$
\mathscr{S}(f)(n)=\max \left\{\sum_{i=1}^{k} f\left(n_{i}\right) \mid \sum_{i=1}^{k} n_{i}=n \text { and } n_{i} \in \mathbb{N}\right\} .
$$

Observe that the subnegative closure is monotone increasing. Also if $f$ is bounded above by a linear (or quadratic, etc) function then so is $\mathscr{S}(f)$. Thus in a sense it suffices to study the subnegative closure of the Dehn function.

We associate to any acceptable extension $\mathscr{E}: A \subset B \rightarrow B / / A$ a function $\alpha_{\mathscr{C}}: \mathbb{N} \rightarrow \mathbb{N}$ and to any complex $X$ another function $\beta_{X}: \mathbb{N} \rightarrow$ $\mathbb{N} \cup\{\infty\}$. These have the property that if $\mathscr{E} \in \mathscr{A}(X)$ then

(1) $\alpha_{\mathscr{E}} \leq \beta_{X}$

(2) $\delta_{B} \preccurlyeq \alpha_{\mathscr{E}}+\mathscr{S}\left(\delta_{A}\right) \circ \alpha_{\mathscr{E}}^{\prime}$, for some function $\alpha_{\mathscr{E}}^{\prime} \equiv \alpha_{\mathscr{C}}$.

$\beta_{X}$ is an upper bound for the combinatorial area of reduced VanKampen diagrams as a function of the length of the boundary cycle. If we are able to compute $\beta_{X}$ then, using the above formulas, we can get an bound for $\delta_{B}$.

We will show that $\beta_{X}=\delta_{X}$ when $X$ is diagramatically reducible and is eventually infinite otherwise. So if $X$ is diagramatically reducible then $\delta_{B} \preccurlyeq \delta_{X}+S\left(\delta_{A}\right) \circ \delta_{X}^{\prime}$ for some function $\delta_{X}^{\prime} \equiv \delta_{X}$. In terms of degrees, we have the more succinct result that $\operatorname{deg}(B) \leq$ $\operatorname{deg}(A) \cdot \operatorname{deg}(X)$. 
We are indebted to Hamish Short for suggesting that we study extensions of two-complexes.

1. The functions $\alpha_{\mathscr{E}}$ and $\beta_{X}$. Suppose $\mathscr{E}: A \subset B \rightarrow B / / A$ is in $\mathscr{A}(X)$. We need to define the functions $\alpha_{\mathscr{E}}$ and $\beta_{X}$. Denote the quotient map $B \rightarrow B / / A$ by $p$.

We start with the definition of $\alpha_{\mathscr{C}}$. Let $w \in N_{B}(l)$. Take $\mathbb{D}=$ $(D, j)$, a Van-Kampen diagram for $w$. Also regard $w$ as a map $j: \partial D \rightarrow B$. Assume that $w$ is not contained entirely in $A$, i.e. $w \notin N_{A}(l)$. The composites $p(w)=p \circ j \uparrow \partial D$ and $p \circ j$ need not be cellular. However, we will describe a method that yields an edge-circuit, $p^{\prime}(w)$, and a diagram $p^{\prime}(\mathbb{D})$ for $p^{\prime}(w)$ where $p^{\prime}(w)$ is homotopic to $p(w)$ in $X^{(1)}$. Begin by identifying each component in $p(w)$ and $D$ of the inverse image (under $p \circ j$ ) of $A$ to a point. Clearly the resulting path, $p^{\prime}(w)$, is an edge-circuit in $X$ with $\left|p^{\prime}(w)\right| \leq|w| \leq l$. But these identifications on $D$ may yield a wedge of a simply-connected planar complex with a collection of two-spheres, wedged together at possibly several different points. The two-spheres result when some loop $u \subset D$ maps into $A$ yet the region in $D$ that $u$ bounds does not. Simply discard any such two-sphere. Call the resulting simply-connected planar two-complex $p^{\prime}(D)$. There is an induced map of $p^{\prime}(D)$ into $B / / A$ with boundary cycle mapping to $p^{\prime}(w)$. We thus have a Van-Kampen diagram for $p^{\prime}(w)$. Denote it by $p^{\prime}(\mathbb{D})$. Note that $a\left(p^{\prime}(\mathbb{D})\right) \leq a(\mathbb{D})$.

If $w \in N_{A}(l) \subset N_{B}(l)$ then it may happen that a minimal VanKampen diagram $\mathbb{D}$ for $w$ does not map entirely into $A$. Of course there are Van-Kampen diagrams for $w$ that map into $A$. But they may have larger area. In any case, the above construction yields the empty diagram for $p^{\prime}(\mathbb{D})$.

Given $l \in \mathbb{N}$, define

$$
W_{\mathscr{E}}(l)=\left\{w \in N_{B}(l) \mid \delta_{B}(l)=\Delta_{B}(w)\right\} .
$$

A $\delta_{B}(l)$-diagram is a minimal Van-Kampen diagram of some $w \in$ $W_{\mathscr{E}}(l)$. Define, for $l \in \mathbb{N}$,

$$
\alpha_{\mathscr{C}}(l)=\min \left\{a\left(p^{\prime}(\mathbb{D})\right) \mid \mathbb{D} \text { is a } \delta_{B}(l) \text {-diagram }\right\}
$$

(if it happens that some $\delta_{B}(l)$-diagram or some $w \in W_{\mathscr{E}}(l)$ maps into $A$ then $\left.\alpha_{\mathscr{E}}(l)=0\right)$.

We turn to the function $\beta_{X}$. Given a Van-Kampen diagram $(D, j)$ a pair of distinct faces $F$ and $F^{\prime}$ of $D$ are opposite under $j$ if $F \cap F^{\prime}$ contains at least an edge and there is an orientation reversing 
homeomorphism $g: F^{\prime} \rightarrow F$, fixing $F \cap F^{\prime}$ pointwise, with $j \circ g$ । $F^{\prime}=j \uparrow F$. In other words the map $j$ "folds" the faces across their intersection. Note it is often difficult determining whether two faces are opposite. One must consider the whole of each face and not just the boundary, especially when the image of the boundary involves a repetition of edges. A Van-Kampen diagram is reduced if it has no opposite faces. A cut-and-paste argument shows that if $w \in N_{X}$ then there is a reduced Van-Kampen diagram for $w$. The cut-and-paste operation may yield a wedge of planar complex together with a sphere. One discards any such sphere. And as the cut-and-paste operation decreases $a(\mathbb{D})$, we see that any minimal Van-Kampen diagram must be reduced.

Given $w \in N_{X}(l)$, let

$$
\nabla_{X}(w)=\sup \{a(\mathbb{D}) \mid \mathbb{D} \text { is a reduced diagram for } w\}
$$

and define

$$
\beta_{X}(l)=\sup \left\{\nabla_{X}(w) \mid w \in N_{X}(l)\right\} .
$$

Compare these formulas with those defining $\Delta_{K}$ and $\delta_{K}$ in $\S 0$. Note that $\beta_{X}$ is also given by the expression

$$
\beta_{X}(l)=\sup \{a(\mathbb{D}) \mid \mathbb{D} \text { is a reduced diagram }
$$

$$
\text { mapping into } X \text { with }|\partial \mathbb{D}| \leq l\} \text {. }
$$

Thus $\beta_{X}$ gives an upper bound for the area of a reduced diagram as a function of the length of the boundary cycle. The function $\beta_{X}$ may be infinite-valued. Consider, for example the two-sphere $S$ with two vertices, $v_{0}$ and $v_{1}$, one edge, $e$, from $v_{0}$ to $v_{1}$, and one face, attached according to the word $e e^{-1}$. Then $\beta_{S}(l)=\infty$ for all $l \geq 2$ as can be seen from the reduced Van-Kampen diagram for the word $e e^{-1}$ pictured in Figure 1.1 (this diagram also illustrates the subtlety of deciding whether or not a diagram is reduced). Here, once orientations

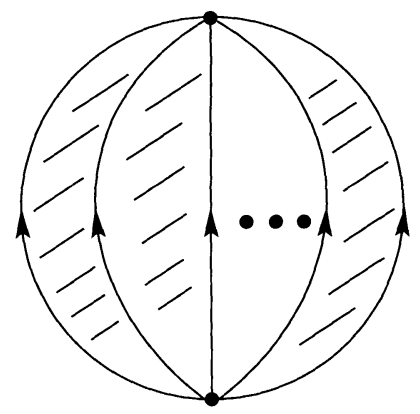

Figure 1.1 
for the diagram and the two-sphere have been fixed, the faces of the diagram all map in the same way.

The relation between $\alpha_{\mathscr{E}}$ and $\beta_{X}$ is found in the result:

Proposition 1.1. If $\mathscr{E} \in \mathscr{A}(X)$ then $\alpha_{\mathscr{E}} \leq \beta_{X}$.

Proof. Write $\mathscr{E}: A \subset B \rightarrow X$ and $p: B \rightarrow X$ the quotient map. Fix $l \in \mathbb{N}$ and suppose $\mathbb{D}$ is a $\delta_{B}(l)$-diagram. Since $\mathbb{D}$ is a minimal diagram, it must be reduced. And since $X$ has no redundant faces, the induced Van-Kampen diagram $p^{\prime}(\mathbb{D})$ is also reduced. Since $\left|\partial p^{\prime}(\mathbb{D})\right| \leq|\partial \mathbb{D}| \leq l$ we have $\alpha_{\mathscr{E}}(l) \leq a\left(p^{\prime}(\mathbb{D})\right) \leq \beta_{X}(|\partial \mathbb{D}|) \leq \beta_{X}(l)$.

2. Extensions. Given an extension $\mathscr{E}: A \subset B \rightarrow B / / A$ in $\mathscr{A}(X)$, we want to use the function $\alpha_{\mathscr{E}}$ to obtain an upper bound for $\delta_{B}$. The key point is that $\alpha_{\mathscr{E}}$ tells us how a $\delta_{B}(l)$-diagram "projects" to $X$. It is then a matter of seeing how it pulls back.

Proposition 2.1. If $\mathscr{E}: A \subset B \rightarrow B / / A$ is in $\mathscr{A}(X)$ then there is some function $\alpha_{\mathscr{E}}^{\prime} \equiv \alpha_{\mathscr{E}}$ with $\delta_{B}(l) \leq \alpha_{\mathscr{E}}(l)+\mathscr{S}\left(\delta_{A}\right) \circ \alpha_{\mathscr{E}}^{\prime}(l)$ for all $l \in \mathbb{N}$.

Proof. Fix $l \in \mathbb{N}$ and let $\mathbb{D}=(D, j)$ be a $\delta_{B}(l)$-diagram for $w$ with $\alpha_{\mathscr{E}}(l)=a\left(p^{\prime}(\mathbb{D})\right.$ ) (where $p: B \rightarrow X$ is the quotient map, and $p^{\prime}$ is defined in $\S 1)$.

Suppose there is some loop $u \subset D$ that maps into $A$ but the region, $D_{u}$, in $D$ that $u$ bounds does not. Since $\mathscr{E} \in \mathscr{A}(X), A$ is $\pi_{1}$ injective in $B$. So there is some minimal diagram $\left(D_{u}^{\prime}, j\right)$ for $u$ mapping into $A$ (minimal in $A$ ), though with possibly larger area than $D_{u}$. Cut $D_{u}$ from $D$ and replace it with $D_{u}^{\prime}$. Do this for each such loop $u$. We will abuse notation and also call the resulting diagram $\mathbb{D}$. Observe that we may have increased the area of $\mathbb{D}$, but the diagram $p^{\prime}(\mathbb{D})$ is unchanged. So $\delta_{B}(l) \leq a(\mathbb{D})$ and $\alpha_{\mathscr{E}}(l)=a\left(p^{\prime}(\mathbb{D})\right)$.

$p^{\prime}(\mathbb{D})$ and $p^{\prime}(w)$ differ from $\mathbb{D}$ and $w$ only in that certain subcomplexes of $\mathbb{D}$ and subpaths of $w$-those that mapped into $A$-have been replaced by vertices. Call these subcomplexes the $A$-subcomplexes of $\mathbb{D}$, the subpaths the $A$-subpaths of $w$, and the vertices that replace either of them the $A$-vertices. We need to count the areas of the $A$-subcomplexes.

Each two-cell $E^{\prime}$ of $X$ corresponds to some two-cell $E$ of $B$. The attaching map of $E^{\prime}$ differs from that of $E$ by the deletion of any word mapping to $A^{(1)}$. Let $K$ be the maximum of the lengths of any such deleted word for any two-cell of $X$. This bound is finite since 

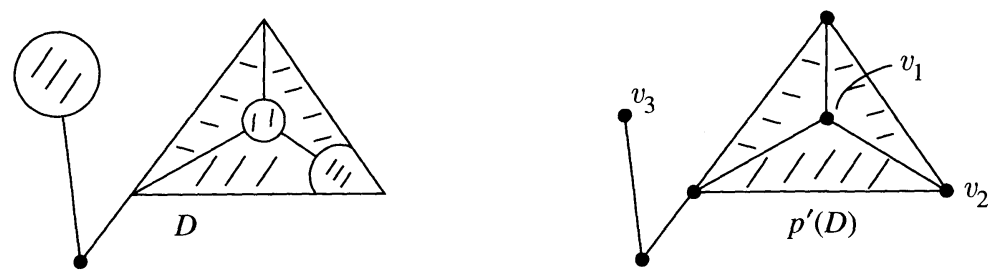

FIGURE 2.1

$X$ has only finitely many two-cells. There is also a bound $K^{\prime}$ to the length of the boundaries of the two-cells of $X$. Let $K^{\prime \prime}=2 \cdot K \cdot K^{\prime}$. We will show that

$$
a(\mathbb{D}) \leq \alpha_{\mathscr{E}}(l)+\mathscr{S}\left(\delta_{A}\right)\left(l+K^{\prime \prime} \alpha_{\mathscr{E}}(l)\right) .
$$

Note that the function $\alpha_{\mathscr{E}}^{\prime}(l)=l+K^{\prime \prime} \cdot\left(\alpha_{\mathscr{E}}(l)\right)$ is $\equiv$ to $\alpha_{\mathscr{E}}$.

The $A$-vertices are of three different types:

(1) those that are interior vertices of $p^{\prime}(D)$,

(2) those that are on the boundary of some subdisc of $p^{\prime}(D)$, and

(3) those with one-dimensional neighborhoods.

These conditions partition the set of $A$-vertices into subsets $V_{1}, V_{2}$, and $V_{3}$. See Figure 2.1 (where $v_{i} \in V_{i}$ ).

Suppose $v \in V_{1}$ replaces the $A$-subcomplex $S_{v}$. Let $\gamma_{v}$ be the link of $v$ in $p^{\prime}(D)$. This loop can be viewed as corresponding to the boundary cycle $\partial S_{v}$ of $S_{v}$ in $D$. Denote the index of the vertex $v$ in $D$ by $i(v)$. Then clearly, we have that $\left|\partial S_{v}\right| \leq K \cdot i(v)$. And thus, since $\mathbb{D}$ is a minimal diagram,

$$
a\left(S_{v}\right) \leq \delta_{A}(K \cdot i(v)) .
$$

Now suppose $v$ is a boundary $A$-vertex. Let $w_{v}$ be the $A$-subpath of $w$ that $v$ replaces (of course $w_{v}$ may itself just be a vertex). Assume that $v \in V_{2}$ and let $v$ correspond to the $A$-subcomplex $S_{v}^{\prime}$. Then, as above, the boundary cycle of $S_{v}^{\prime}$ can be viewed as corresponding to the concatenation of components of the link of $w$ in $D$ with the subpath $w_{v}$. And so $\left|\partial S_{v}^{\prime}\right| \leq\left|w_{v}\right|+K \cdot i(v)$. Since $\mathbb{D}$ is minimal we have that

$$
a\left(S_{v}^{\prime}\right) \leq \delta_{A}\left(\left|w_{v}\right|+K \cdot i(v)\right) .
$$

Assume now that $v \in V_{3}$ and let $w_{v}$ be the $A$-subpath of $w$ that $v$ replaces. Denote by $S_{v}^{\prime \prime}$ the $A$-subcomplex that $v$ replaces. Then the boundary cycle of $S_{v}^{\prime \prime}$ corresponds to the subpath $w_{v}$. Hence

$$
a\left(S_{v}^{\prime \prime}\right) \leq \delta_{A}\left(\left|w_{v}\right|\right) \text {. }
$$


So we have three types of $A$-components. Adding their areas together with the area of $p^{\prime}(\mathbb{D})$ yields the area of $\mathbb{D}$. The above formulas yield:

$$
\begin{aligned}
a(\mathbb{D}) \leq & a\left(p^{\prime}(\mathbb{D})\right)+\sum_{v \in V_{1}} \delta_{A}(K \cdot i(v)) \\
& +\sum_{v \in V_{2}} \delta_{A}\left(\left|w_{v}\right|+K \cdot i(v)\right)+\sum_{v \in V_{3}} \delta_{A}\left(\left|w_{v}\right|\right) .
\end{aligned}
$$

Let $\sum_{v \in V_{1} \cup V_{2}} i(v)=N$ and $\sum_{v \in V_{2} \cup V_{3}}\left|w_{v}\right|=M$. Using subnegativity gives

$$
a(\mathbb{D}) \leq a\left(p^{\prime}(\mathbb{D})\right)+\mathscr{S}\left(\delta_{A}\right)(M+K N) .
$$

Since the $A$-subpaths are disjoint we have $M \leq|w| \leq l$. Regarding $N$, it is bounded above by twice the number of edges of $p^{\prime}(D)$. The number of edges of $p^{\prime}(D)$ is bounded above by $K^{\prime}$. (the number of faces of $\left.p^{\prime}(D)\right)$. And the number of faces is $\alpha_{\mathscr{E}}(l)$. Thus $N \leq$ $2 \cdot K^{\prime} \alpha_{\mathscr{E}}(l)$. Putting this together (using $K^{\prime \prime}=2 K K^{\prime}$ and the fact that $\mathscr{S}\left(\delta_{A}\right)$ is monotone increasing)

$$
\delta_{B}(l) \leq a(\mathbb{D}) \leq \alpha_{\mathscr{E}}(l)+\mathscr{S}\left(\delta_{A}\right)\left(l+K^{\prime \prime} \alpha_{\mathscr{E}}(l)\right)
$$

as desired.

By Proposition 1.1 and the preceding, we see that knowing $\beta_{X}$ allows us to bound $\delta_{A}$. In the final two sections we will compute $\beta_{X}$.

3. Non-DR complexes. Recall from [Ge1] that a two-complex $X$ is diagramatically reducible (abbreviated DR) if any combinatorial map of a two-sphere into $X$ has a pair of opposite faces. Some examples of DR complexes are collapsible complexes and hyperbolic surfaces.

In this section we will show that $\beta_{X}$ is eventually infinite when $X$ fails to be DR.

We need two facts for our result:

(1) the composition of reduced maps is reduced,

(2) if $X$ is the two-sphere then $\beta_{X}$ is eventually infinite.

The first fact follows trivially from the definition of a reduced map. The second comes from a generalization of the example given in $\S 1$. and pictured in Figure 1.1. If $X$ is any cell structure of the twosphere, that has more than a single two-cell, then one can construct similar reduced Van Kampen diagrams by letting $e$ be any edge of $X$ that appears in exactly two faces of $X$ (so what is pictured as a single two-cell in Figure 1.1 is replaced by a complex homeomorphic to $X$ cut open along $e$ ). We thus see that $\beta_{X}(l)=\infty$ for $l \geq 2$. 
Using these facts, we obtain our result on $\beta_{X}$ for non-DR complexes:

Proposition 3.1. Suppose $X$ is a two-complex that is not diagramatically reducible. Then $\beta_{X}$ is eventually infinite.

Proof. Let $\phi: T \rightarrow X$ be a reduced map of a two-sphere. We know from above that $\beta_{T}$ is eventually infinite. Let $m \geq 2$. Given an integer $k$, there is a reduced Van-Kampen diagram $j: D \rightarrow T$, having boundary cycle length $\leq m$, with area greater than $k$. Then the composition $\phi \circ j: D \rightarrow X$ is reduced, by the above, and has area greater than $k$. As $k$ was arbitrary, we get $\beta_{X}(m)=\infty$.

4. DR complexes. We will show in this section that $\beta_{X}=\delta_{X}$ when $X$ is DR.

We define a sphere-graph complex to be a union of two-spheres together with some graphs glued together at vertices. Suppose $\phi: L \rightarrow X$ is a combinatorial map of a sphere-graph complex. Let $F$ and $F^{\prime}$ be a pair of opposite faces for $\phi$. One can cut out $F$ and $F^{\prime}$, identifying $\partial F$ and $\partial F^{\prime}$, using the orientation reversing homeomorphism $g$, yielding a combinatorial map $\bar{\phi}: \bar{L} \rightarrow X$ of a new sphere-graph complex. The faces of $\bar{L}$ can be identified with the faces of $L \backslash\left\{F, F^{\prime}\right\}$.

Assume that each two-sphere in $L$ is partitioned into "upper" and "lower" hemispheres. Note that each two-sphere of $\bar{L}$ inherits such a partition. Now assume that $F$ lies in some upper hemisphere while $F^{\prime}$ lies in some lower hemisphere, where $F$ and $F^{\prime}$ are opposite under $\phi$. If $E$ and $E^{\prime}$ are opposite faces under $\bar{\phi}$, both lying in upper hemispheres then the corresponding faces of $L$ are opposite under $\bar{\phi}$. Similarly for faces both lying in lower hemispheres.

We will use this in the following:

Proposition 4.1. Suppose $X$ is diagramatically reducible. Then

$$
\beta_{X}=\delta_{X}
$$

Proof. Suppose $w$ is an inessential edge-circuit in $X$. If $j_{1}: D_{1} \rightarrow$ $X$ and $j_{2}: D_{2} \rightarrow X$ are reduced Van-Kampen diagrams that $w$ bounds then glue them together along their boundary cycles yielding $\phi: L \rightarrow$ $X$. Partition $L$ into upper hemispheres $D_{1}$ and lower hemispheres $D_{2}$. Now $\phi$ is a combinatorial map of a sphere-graph complex into the DR complex $X$. Hence $\phi$ has a pair of opposite faces. Moreover, since $j_{1}$ and $j_{2}$ were assumed to be reduced, the pair of opposite 
faces must lie in different hemispheres. A cut and paste operation as described above yields a combinatorial map $\bar{\phi}: \bar{L} \rightarrow X$ of a new sphere-graph complex. This complex is likewise partitioned into upper and lower hemispheres and has a smaller number of faces. Again, since $X$ is DR, the map $\bar{\phi}$ must have a pair of opposite faces. And, by the comments above, the pair of opposite faces cannot both be upper (or lower) hemispheres. In other words, one of the opposite faces corresponds to a face in $D_{1}$, and the other a face in $D_{2}$.

We then proceed by induction on the number of faces. The end result is a bijection between the faces of $D_{1}$ and the faces of $D_{2}$. Hence the areas of $D_{1}$ and $D_{2}$ are the same

We thus see that any two reduced Van-Kampen diagrams have the same area. Our result then follows.

Note that the above proof can be interpreted as saying that Plateau's problem and the minimal area problem for a DR complex have the same solution.

Combining the preceding proposition with Proposition 2.1 yields:

Corollary 4.2. Suppose $A \subset B$ are a pair of two-complexes with combinatorial quotient $X$ being diagramatically reducible. Then

$$
\delta_{B} \preccurlyeq \delta_{X}+S\left(\delta_{A}\right) \circ \delta_{X}^{\prime}
$$

for some function $\delta_{X}^{\prime} \equiv \delta_{X}$. And if $A$ is two-dimensional then

$$
\delta_{B} \preccurlyeq S\left(\delta_{A}\right) \circ \delta_{X}^{\prime}
$$

Proof. We need only observe that any extension $A \subset B \rightarrow X$ is acceptable, i.e. that $X$ has no redundant faces and that $A \subset B$ is $\pi_{1}$-injective. The former follows from the facts that the two-sphere is not DR and a subcomplex of a DR complex is DR. The latter follows from the fact that a DR complex is Kervaire and thus $G$-Kervaire (see [Br2]).

Since taking subnegative closures does not affect degrees, we have:

Corollary 4.3. Suppose $A \subset B$ are a pair of two-complexes with combinatorial quotient $X$ being diagramatically reducible. Then

$$
\operatorname{deg}\left(\pi_{1}(B)\right) \leq \operatorname{deg}\left(\pi_{1}(A)\right) \cdot \operatorname{deg}\left(\pi_{1}(X)\right) .
$$


5. Examples. We close with a few simple examples (which can be generalized in obvious ways). The first two make use of two theorems of Gromov's (see [Gr]):

(1) if $G$ is hyperbolic then it does not contain $\mathbb{Z} \oplus \mathbb{Z}$ as a subgroup,

(2) if the Dehn function of $G$ has subquadratic growth then $G$ is hyperbolic.

In fact, we will use a strengthened version of the second result (see [Ol]):

$\left(2^{\prime}\right)$ if $G$ is not hyperbolic then its Dehn function is at least quadratic.

Thus a group that contains $\mathbb{Z} \oplus \mathbb{Z}$ as a subgroup has Dehn function at least a quadratic.

If $A$ is a set, write $W(A)$ for the semigroup of (unreduced) words in the alphabet $A$. Consider the group $G$ with presentation

$$
\langle a, b, c, d, x, y \mid w,[x, y]\rangle,
$$

where the natural retraction,

$$
W(\{a, b, c, d, x, y\}) \rightarrow W(\{a, b, c, d\}),
$$

sends $w$ to $[a, b][c, d]$ (note that no free reductions are allowed). Writing $B$ for the two-complex associated to this presentation, we see that $B$ is an extension

$$
A \subset B \rightarrow B / / A
$$

where $A$ is two-complex associated to the presentation

$$
\langle x, y \mid[x, y]\rangle \text {. }
$$

The quotient $B / / A$ is the two-complex associated to

$$
\langle a, b, c, d \mid[a, b][c, d]\rangle
$$

which is a hyperbolic surface, and hence DR (thus $B$ is an extension of a torus by a hyperbolic surface of genus two). Applying Corollary 4.2 , we see that $G$ has Dehn function bounded by a quadratic. Since $G$ contains a copy of $\mathbb{Z} \oplus \mathbb{Z}$, it follows that the Dehn function of $G$ is of quadratic type.

In a similar vein, one can show that the group having presentation

$$
\langle x, y, a, b, c, d \mid[x w, y u],[a, b][c, d]\rangle,
$$

where $w$ and $u$ are in $W(\{a, b, c, d\})$, also has Dehn function of quadratic type. Here we take $A$ to be the two-complex associated to the presentation

$$
\langle a, b, c, d \mid[a, b][c, d]\rangle
$$


and then the quotient complex is

$$
\langle x, y \mid[x, y]\rangle \text {. }
$$

Thus we have an extension of a hyperbolic surface of genus two by a torus. This yields a quadratic upper bound. And, as above, Gromov's theorems give a quadratic lower bound.

Now let $G$ have presentation

$$
\langle a, b, c, d, t \mid w\rangle
$$

where $w$ is a word in $W(\{a, b, c, d, t\})$ that is mapped to $[a, b][c, d]$ by the natural retraction

$$
W(\{a, b, c, d, t\}) \rightarrow W(\{a, b, c, d\}) .
$$

Take $A$ to be the complex associated to $\langle t \mid\rangle$, i.e. a circle. The quotient complex is a hyperbolic surface of genus two. An application of our results show that $G$ is hyperbolic.

In closing, it is worth pointing out here that our theorem does not apply naturally to most group-theoretic extensions. For example, consider the Baumslag-Solitar group given by the presentation

$$
\left\langle a, b \mid a b a^{-1}=b^{2}\right\rangle
$$

has Dehn function of exponential type (see [Ge2]). The group is also an extension of $\mathbb{Z}$ by $\mathbb{Z}$. However expressing the associated twocomplex as a combinatorial extension in the "natural" way yields quotient complex being the two-sphere (the complex associated to $\left.\left\langle a \mid a a^{-1}\right\rangle\right)$ which is not DR.

\section{REFERENCES}

[A1] J. M. Alonso, Inegalités isopérimétriques et quasi-isométries, C.R. Acad. Sci., 311 Serie 1 (1991), 761-764.

[Br1] S. G. Brick, Dehn functions and products of groups, to appear, Trans. Amer. Math. Soc., 335 (1993), 369-384.

[Br2] $\quad$ A note on coverings and Kervaire complexes, Bull. Austral. Math. Soc., 46 (1992), 1-21.

[CEHLPT] J. W. Cannon, D. B. A. Epstein, D. F. Holt, S. V. F. Levy, M. S. Paterson, and W. P. Thurston, Word processing in groups, Jones and Bartlett, 1992:

[Ge1] S. M. Gersten, Reducible diagrams and equations over groups, Essays in group theory (MSRI Publ., Vol. 8), Springer, Berlin-Heidelberg-New York, 1987, pp. 15-74.

[Ge2] S. M. Gersten, Dehn functions and $l_{1}$-norms of finite presentations, Algorithms and Classification in Combinatorial Group Theory (G. Baumslag and C. F. Miller III, eds.), M.S.R.I. series, vol. 23, Springer-Verlag, 1991. 
M. Gromov, Hyperbolic groups, Essays in Group Theory (S. M. Gersten, ed.), M.S.R.I. series, vol. 8, Springer Verlag, 1987.

[Ol] A.Y. Ol'shanskii, Hyperbolicity of groups with subquadratic isoperimetric inequality, IJAC, 1 (1991), 281-289.

Received June 15, 1991 and in revised form May 17, 1992.

DEPARTMENT OF MATHEMATICS

UNIVERSITY OF OKLAHOMA

NORMAN, OK 73019

Current address: Department of Mathematics

University of California-Davis

Davis, CA 95616

E-mail address: brick@ucdmath.ucdavis.edu 



\title{
PACIFIC JOURNAL OF MATHEMATICS
}

Founded by

\author{
E. F. BECKENBACH (1906-1982) F. WolF (1904-1989)
}

\section{EDITORS}

Sun-Yung A. Chang

(Managing Editor)

University of California

Los Angeles, CA 90024-1555

chang@math.ucla.edu

\section{F. Michael Christ}

University of California

Los Angeles, CA 90024-1555

christ@math.ucla.edu

HeRbert Clemens

University of Utah

Salt Lake City, UT 84112

clemens@math.utah.edu
Thomas ENRIGHT

University of California, San Diego

La Jolla, CA 92093

tenright@ucsd.edu

Nicholas ERCOLANI

University of Arizona

Tucson, AZ 85721

ercolani@math.arizona.edu

\section{R. FINN}

Stanford University

Stanford, CA 94305

finn@gauss.stanford.edu

VAughan F. R. Jones

University of California

Berkeley, CA 94720

vfr@math.berkeley.edu
SteVen KerckhofF

Stanford University

Stanford, CA 94305

spk@gauss.stanford.edu

Martin ScharlemanN University of California Santa Barbara, CA 93106 mgscharl@henri.ucsb.edu

\section{Harold Stark}

University of California, San Diego La Jolla, CA 92093

V. S. VARADARAJAN University of California Los Angeles, CA 90024-1555 vsv@math.ucla.edu

\section{SUPPORTING INSTITUTIONS}

UNIVERSITY OF ARIZONA

UNIVERSITY OF BRITISH COLUMBIA

CALIFORNIA INSTITUTE OF TECHNOLOGY

UNIVERSITY OF CALIFORNIA

UNIVERSITY OF MONTANA

UNIVERSITY OF NEVADA, RENO

NEW MEXICO STATE UNIVERSITY

OREGON STATE UNIVERSITY
UNIVERSITY OF OREGON

UNIVERSITY OF SOUTHERN CALIFORNIA

STANFORD UNIVERSITY

UNIVERSITY OF HAWAII

UNIVERSITY OF UTAH

WASHINGTON STATE UNIVERSITY

UNIVERSITY OF WASHINGTON 


\section{PACIFIC JOURNAL OF MATHEMATICS}

Volume $161 \quad$ No. $1 \quad$ November 1993

Tangential and normal Euler numbers, complex points, and

singularities of projections for oriented surfaces in four-space

ThOMAS FRANCIS BANCHOFF and FrANK ALBERT FARRIS

Studying links via closed braids. III. Classifying links which are closed 3-braids

JOAN Birman and William W. MEnAsCo

Dehn functions of groups and extensions of complexes

STEPHEN GARY BRICK

Contact structures on $(n-1)$-connected $(2 n+1)$-manifolds

\section{HANSJÖRG GEIGES}

The relative Nielsen number and boundary-preserving surface maps

MiCHAEL R. KELLY

Besov spaces, mean oscillation, and generalized Hankel operators

\section{MARCo Maria PELOSO}

On the nonoccurrence of the Coxeter graphs $\beta_{2 n+1}, D_{2 n+1}$ and $E_{7}$ as 185 the principal graph of an inclusion of $\mathrm{II}_{1}$ factors

ViakALATHUR SHANKAR SUNDER and A. K. ViJAYARAJAN 